\title{
Die Weiterbildung in psychoanalytisch-systemischer Psychotherapie für Kinder, Jugendliche und Familien am Institut KJF in Luzern
}

\author{
Ein Ein- und Überblick
}

Roland Müller (Luzern)

Das Institut für Kinder-, Jugendlichen- und Familientherapie $\left(\mathrm{KJF}^{1}\right)$ ist 1997 in Luzern entstanden. Sieben PsychotherapeutInnen und Kinder- und JugendpsychiaterInnen mit psychoanalytischer Orientierung sowie ein administrativer Leiter gründeten einen Trägerverein und entwarfen ein vierjähriges Curriculum für eine Psychotherapie-Weiterbildung auf psychoanalytisch-systemischer Grundlage.

Nach rund drei-jähriger Vorbereitung startete im August 2000 ein erster Weiterbildungskurs von 4 Jahren mit 24 Teilnehmenden (vorwiegend mit Ausbildung in Medizin und Psychologie). Bis heute konnten drei Weiterbildungskurse mit je ca. 20 Teilnehmenden abgeschlossen werden; ein vierter Kurs läuft seit August 2007, und ein fünfter wird im Januar 2010 beginnen. Für diesen fünften Kurs wurde erstmals in enger Zusammenarbeit mit der EFPP Deutsche Schweiz (Sektion Psychoanalytische Kinder- und Jugendlichenpsychotherapie) ein einjähriger Vorkurs (mit Schwerpunkt Säuglings- und Kleinkinderbeobachtung sowie Beginn einer eigenen hochfrequenten Analyse) angeboten und im September 2008 gestartet, womit Teilnehmenden das Erreichen der europäischen EFPP-Standards für die Zertifizierung als psychoanalytische/-r Kinder- und JugendlichenpsychotherapeutIn ermöglicht werden soll.

\section{Die Entstehung}

Hintergrund der KJF-Gründung ist einerseits die Entstehung des Psychoanalytischen Seminars in Luzern (PSL ${ }^{2}$ ) im Jahre 1992, welches von PSZTeilnehmerInnen nach ihrer Weiterbildung in Zürich gegründet wurde, um eine kontinuierliche Möglichkeit der Auseinandersetzung mit der Freudschen Psychoanalyse auch in Luzern zu schaffen. Das PSL versteht sich in diesem Sinne nicht als Weiterbildungsinstitut wie das PSZ, sondern als Fortbildungsinstitut.

Unter den anfangs gegen hundert Teilnehmenden des PSL befanden sich sehr viele psychoanalytisch orientierte Kinder- und JugendlichentherapeutInnen 
(häufig mit PSZ-Weiterbildung), so dass ein Interesse an entsprechenden Seminaren, Kursen und Supervisionen auch im PSL aufkam - und über dieses hinaus dann die Idee, in Luzern eine Weiterbildung in Psychoanalytischer Kinderund Jugendlichentherapie auf die Beine zu stellen.

Anderseits waren gerade solche Projekte innerhalb des PSZ zu Beginn der 90er Jahre von der dortigen «Arbeitsgruppe Kinderpsychoanalyse» (mit ihrem «Förderverein Kinderpsychoanalyse» als geplantem finanziellem Background) verfolgt worden. Zu erinnern ist an die beiden erfolgreichen KinderpsychoanalyseSymposien 1992 und 1994 am PSZ. Leider scheiterte aber das Bemühen dieser Gruppe jüngerer Kinder- und JugendlichenanalytikerInnen in einer damals institutionalisierungskritischen Atmosphäre, eine «Ausbildung» (seit Bologna: «Weiterbildung») in Psychoanalytischer Kinder- und Jugendlichenpsychotherapie im PSZ aufzubauen.

Auf diesem Hintergrund entstand im PSL - neben zahlreichen Seminaren und Lesegruppen zu Themen der Kinder- und Jugendlichenanalyse - eine Fortbildung in «Psychoanalytischer Familientherapie» mit Prof. Michael Buchholz (Göttingen). Zunächst in Form von Vorträgen und Supervisionen, anschliessend mit drei je zweijährigen Kursen in Psychoanaytischer Familientherapie bei Michael Buchholz, an welchen auch viele vom PSZ teilnahmen.

Gemeinsam mit Michael Buchholz entstand aus diesen Kursen heraus, und auf dem erwähnten Hintergrund des Scheiterns entsprechender Bemühungen im PSZ, bei einigen AbsolventInnen und auch bei Professor Buchholz die Idee, in Luzern eine neue Weiterbildung aufzubauen. 1997entstand eine Kerngruppe aus sieben psychoanalytisch ausgebildeten und psychoanalytisch orientierten Kolleginnen und Kollegen. Zunächst begleitet von Michael Buchholz als Berater, erarbeiteten sie in einem längeren Prozess ein Curriculum ${ }^{3}$ und gründeten schliesslich 1999 einen Trägerverein.

\section{Das Ziel}

Hauptziel des KJF ist es, eine theoretisch wie praktisch kompakte vierjährige «psychoanalytisch-systemische» Weiterbildung von PraktikerInnen für PraktikerInnen anzubieten und damit die Grundlage für eine selbständige psychoanalytisch-psychotherapeutische Tätigkeit sowie die kontinuierliche Fortbildung in diesem Gebiet - u. a. am PSZ - zu schaffen. Wir verstehen uns nicht als Konkurrenz zu anderen Weiterbildungen auf diesem Gebiet, wie etwa der kürzlich entstandenen Weiterbildung für Psychoanalytische Kinder- und Jugendlichenpsychotherapie am PSZ oder in Basel (Prof. Bürgin), sondern als Alternative. 


\section{Das Weiterbildungskonzept}

Mit einem «schulischen» Curriculum sollen die Teilnehmenden auf ihre praktische Tätigkeit in Institutionen der Kinder- und Jugendpsychiatrie, mit diesen verbundenen Institutionen oder der freien Praxis vorbereitet werden. Das Weiterbildungskonzept umfasst hierbei die 5 folgenden Säulen:

, eigene psychoanalytisch orientierte Selbsterfahrung,

, Supervision (im KJF selber: 100 Gruppensupervisionssitzungen; ausserhalb des KJF individuell ergänzbar),

, Theorie (über 400 Lektionen),

, Training (Kurse für Erstinterviews mit Familien, Eltern und Einzelsetting mit Demonstrationen und Rollenspielen in den Kursen sowie Videoanalysen in den Supervisionen) und

> Beobachtung (Videoanalysen in der Supervision; der Kurs «Säuglings- und Kleinkinderbeobachtung» («Baby Observation») während eines Jahres, mit 14-täglichen Familienbesuchen).

\section{Die Ausrichtung}

Inhaltlich orientiert sich die Weiterbildung einerseits an der psychoanalytischen Familientherapie, wie sie in Europa vor allem durch Michael Buchholz definiert wurde. Diese umfasst viele Elemente der Systemtherapie (etwa deren Fragetechnik und das zirkulär-dialektische Denken), die ja mehrheitlich von «verlorenen Töchtern und Söhnen» der Psychoanalyse entwickelt worden war und die nach seiner und unserer Ansicht zahllose ungeborgene Schätze beinhaltet, die auch für eine psychoanalytisch orientierte Arbeit mit Familien und Eltern fruchtbar gemacht werden können. Fürstenau hatte dafür einmal die Losung «Psychoanalytisch verstehen, systemisch denken, suggestiv (für uns lieber: «hilfreich») handeln» geprägt.

Diese Kombination der psychoanalytischen Kinder- und Jugendlichen psychotherapie mit der Familientherapie ist eine wesentliche Komponente unseres inhaltlichen Konzepts und ein Hauptgrund für die von uns gewählte Bezeichnung "psychoanalytisch-systemisch».

Anderseits bezieht sich diese Bezeichnung «psychoanalytisch-systemisch» auf eine Entwicklungsströmung der Psychoanalyse selbst: wir verstehen darunter die konsequente Bezugnahme auf das interaktionelle Feld (z. B. v. Blarer, Stolorow u. a.), eine «Zwei-Personen-Psychologie» (Rickman, Balint), «Übergangsphänomene» (Winnicott), Bindungsmuster (Bowlby) oder die «relationale Matrix» (Mitchell). ${ }^{4}$ Diese «relationale» Ausrichtung wird auch durch die Resultate der psychoanaly- 
tisch orientierten Säuglings- und Bindungsforschung (v.a. Beebe \& Lichtenberg, Stern, «Process of Change Study Group» Boston, Fonagy, Target, Gergely, u.a.) gestützt und gestattet eine integrative, schulenübergreifende Konzeption der Psychoanalyse - mit praktischem Nutzen: der Deutungsprozess wird «demokratisiert», wie es für uns gerade in der Kinder- und Jugendpsychoanalyse Sinn macht. Der Schweizer Kinderanalytiker Hans Zulliger - unterschätzt und heute zu Unrecht vergessen - kann ebenso als früher Wegbereiter dieses Ansatzes in der Kinder- und Jugendlichenpsychoanalyse gesehen werden wie Ferenczi in der Erwachsenenanalyse.

\section{Anmerkungen}

$1 \quad$ Website des KJF: www.institut-kjf.ch

2 Website des PSL: www.psychoanalyse-luzern.ch

3 Heutige Fassung des Curriculums und Programms: siehe unter www.institut-kjf.ch/ $\mathrm{cms} /$ media.php?id=31\&cat=

$4 \quad$ Siehe R. Müller: Psychoanalytische Therapie heute. Auf dem Weg zu neuen Konzeptionen ihrer Praxis. «Journal», Juli 1998, Heft 35, S. 3-13. 